\title{
Performance of a Heuristic Total Weight in Combinatorial Mixture Packaging of Two Types of Items
}

\author{
Yoshiyuki Karuno $^{\mathrm{a}}$ and Oki Nakahama ${ }^{\mathrm{b}}$ \\ ${ }^{a}$ Faculty of Mechanical Engineering, Kyoto Institute of Technology, Kyoto 606-8585, Japan \\ ${ }^{b}$ Graduate School of Science and Technology, Kyoto Institute of Technology, Kyoto 606-8585, Japan \\ * Corresponding Author: karuno@kit.ac.jp
}

\begin{abstract}
In this paper, a combinatorial optimization model of mixture packaging of two types of items is treated, which arises in actual packing systems, so-called multi-head weighers. The primary objective is to minimize the total weight of chosen items for a package under the condition that the total weight must be no less than a specified target weight. For a package to be produced, the weight sum of chosen items of each type must also be no less than a prescribed necessity minimum. In this paper, a heuristic algorithm is designed for the problem of minimizing the total weight, and for a given positive real $\varepsilon$, the performance factor is shown to be $(1+\varepsilon)$.
\end{abstract}

Keywords: Engineering optimization, Combinatorial food packaging, Net weights, Approximation algorithms, Performance guarantee.

\section{Introduction}

In this paper, we consider the following 0-1 integer programming problem:

\section{Problem Q}

$$
\begin{aligned}
\text { minimize } & f(x)=\sum_{i=1}^{2} \sum_{k=1}^{n_{i}} w_{i k} x_{i k} \\
\text { subject to } & \sum_{i=1}^{2} \sum_{k=1}^{n_{i}} w_{i k} x_{i k} \geq t, \\
& \sum_{k=1}^{n_{i}} w_{i k} x_{i k} \geq b_{i}, \quad i=1,2, \\
& x_{i k} \in\{0,1\}, i=1,2, k=1,2, \ldots, n_{i},
\end{aligned}
$$

where as the instance,

- $n_{i}$ : The number of given items of type $i$;

- $w_{i k}$ : A positive integer weight of the $k$-th item of type $i$;

- $t$ : A prescribed target weight of a package, which is assumed to be a positive integer;
- $b_{i}$ : A prescribed necessity minimum of the weight sum of chosen items of type $i$ in a package, which is also assumed to be a positive integer

are given, and the solution is a $0-1$ vector

$$
x=\left(x_{1} ; x_{2}\right)=\left(x_{11}, x_{12}, \ldots, x_{1, n_{1}} ; x_{21}, x_{22}, \ldots, x_{2, n_{2}}\right)
$$

of $n=n_{1}+n_{2}$ variables defined to be

$$
x_{i k}= \begin{cases}1 & \text { if the } k \text {-th item of type } i \text { is chosen } \\ 0 & \text { otherwise }\end{cases}
$$

A 0-1 vector $x$ satisfying Eqs. (2)-(4) is referred to as a feasible solution of problem Q. For an instance of problem Q, let $f^{*}$ denote the minimum of the total weight of chosen items in a feasible solution, and let $x^{*}=\left(x_{1}^{*} ; x_{2}^{*}\right)$ denote an optimal solution which is a feasible solution satisfying $f\left(x^{*}\right)=f^{*}$.

Such a combinatorial optimization model as problem $\mathrm{Q}$ arises in automated food packing systems, known as multihead weighers ${ }^{(1,2,3)}$. The food packing system possesses several (typically, around twenty) hoppers with a weighing function, and some amount of food (such as a green pepper, a handful of potato chips, some pieces of candies, and so on) is thrown into each weighing hopper (see Fig. 1). We call the food in each hopper an item. The food packing system chooses some current items from the corresponding weighing hoppers for a package. The resulting empty hoppers are supplied with next new items, and such a packing operation is repeated to produce a large number of food packages one by one. A more practical model involving the durations of remaining items in hoppers can be found in the literature ${ }^{(4)}$, while in this paper, we concentrate our attention on the primary objective of the total weight (equivalently, the surplus over the target weight) of a package from a viewpoint of mathematical interest.

It has already been known that problem Q is NP-hard, and that it can be solved in pseudo-polynomial time ${ }^{(5,6)}$. Recently, it has been shown that a feasible solution whose total weight is at most twice the minimum is obtained by a polynomial 
time greedy heuristic algorithm, when the weights of items are bounded by the necessity minimum of the weight sum of each item type from the above ${ }^{(7)}$. In this paper, we design another heuristic algorithm for the problem of minimizing the total weight of chosen items in a feasible solution, and we also show that for a given positive real $\varepsilon$, the performance factor is at most $(1+\varepsilon)$. The time complexity of the proposed heuristic algorithm is polynomial in $n$ and $1 / \varepsilon$. Such a design manner of heuristic algorithms is known as a technique of polynomial time approximation schemes ${ }^{(8)}$.
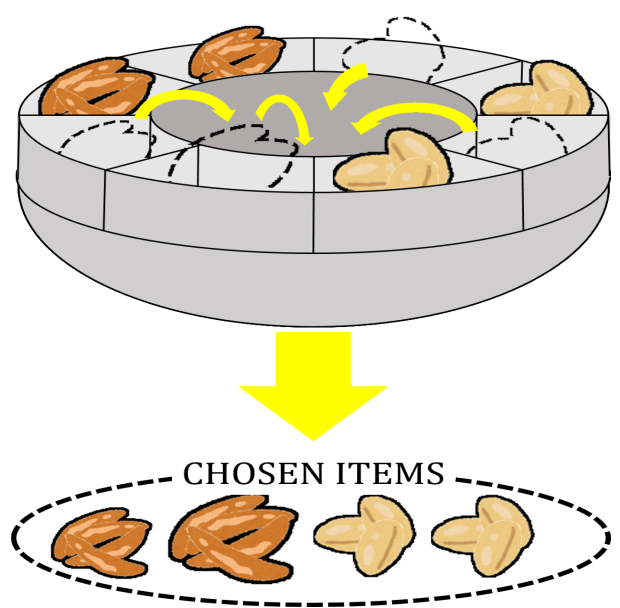

Fig. 1 An illustration of the mixture packing system with $n_{1}=$ $n_{2}=4$, i.e., with $n=8$ weighing hoppers

\section{Preliminaries}

\subsection{Assumptions and Additional Notations}

In order to omit some trivial cases ${ }^{(5,6)}$, we assume that for each type of items,

$$
W_{i}=\sum_{k=1}^{n_{i}} w_{i k} \geq b_{i}, \quad i=1,2,
$$

and also assume that

$$
W=\sum_{i=1}^{2} \sum_{k=1}^{n_{i}} w_{i k} \geq t \geq \sum_{i=1}^{2} b_{i}
$$

We define the maximum of an item weight of each type by

$$
w_{\max }^{(i)}=\max _{1 \leq k \leq n_{i}}\left\{w_{i k}\right\}, \quad i=1,2,
$$

and further assume that

$$
w_{\max }=\max \left\{w_{\max }^{(1)}, w_{\max }^{(2)}\right\}<t .
$$

We are going to consider two partial instances, Q-1 and Q-2, of a given instance of problem $\mathrm{Q}$, which are described as follows:

\section{Problem Q- $i$}

$$
\begin{array}{ll}
\text { minimize } & f_{i}\left(x_{i}\right)=\sum_{k=1}^{n_{i}} w_{i k} x_{i k} \\
\text { subject to } & \sum_{k=1}^{n_{i}} w_{i k} x_{i k} \geq b_{i}, \\
& x_{i k} \in\{0,1\}, k=1,2, \ldots, n_{i} .
\end{array}
$$

A partial solution $x_{i}$ is feasible in the sense of problem Q- $i$ if it satisfies Eqs. (10) and (11). We remark that for a combination $x$ of feasible partial solutions $x_{1}$ and $x_{2}$, $f(x)=f_{1}\left(x_{1}\right)+f_{2}\left(x_{2}\right)<t$ may hold, i.e., the $x$ may be an infeasible solution of problem $\mathrm{Q}$.

Problem Q- $i$ is the minimum subset sum problem ${ }^{(5)}$, and there exists a pseudo-polynomial time dynamic programming procedure ${ }^{(9)}$. By utilizing the dynamic programming procedure, we can easily see that the following property holds:

Lemma 1. For an instance of problem Q- $i$, a maximal subset $X_{i}$ of feasible solutions of the problem such that any two feasible solutions in the $X_{i}$ have different objective function values each other can be obtained in $O\left(n_{i} W_{i}\right)$ time.

\subsection{Rounding Weights of Items}

Let $\delta \geq 1$ denote a positive rounding divisor. The rounded weight of each item is set to be

$$
w_{i k}^{\mathrm{R}}:=\left\lfloor\frac{w_{i k}}{\delta}\right\rfloor \text { for } i=1,2, k=1,2, \ldots, n_{i} .
$$

From the above rounding definition, we have

$$
\begin{aligned}
& \delta w_{i k}^{\mathrm{R}} \leq w_{i k} \text { and } \delta w_{i k}^{\mathrm{R}}>w_{i k}-\delta \\
& \qquad \text { for } i=1,2, k=1,2, \ldots, n_{i} .
\end{aligned}
$$

For a real $\varepsilon>0$, let

$$
\delta=\frac{\varepsilon t}{n} .
$$

Then we provide the following formulation of a rounded instance of a given instance of problem Q:

\section{Problem R}

$$
\begin{aligned}
\text { minimize } & r(x)=\sum_{i=1}^{2} \sum_{k=1}^{n_{i}} w_{i k}^{\mathrm{R}} x_{i k} \\
\text { subject to } & \sum_{i=1}^{2} \sum_{k=1}^{n_{i}} w_{i k} x_{i k} \geq t, \\
& \sum_{k=1}^{n_{i}} w_{i k} x_{i k} \geq b_{i} \quad i=1,2, \\
& x_{i k} \in\{0,1\}, i=1,2, k=1,2, \ldots, n_{i} .
\end{aligned}
$$

A 0 - 1 vector $x=\left(x_{1} ; x_{2}\right)$ satisfying Eqs. (16)-(18) is feasible in the sense of problem R. Notice that two problems $Q$ and $\mathrm{R}$ have the same constraints, and hence a feasible solution 
of problem $\mathrm{Q}$ is also a feasible solution of problem $\mathrm{R}$, and vice versa. For an instance of problem R, let $r^{*}$ denote the minimum of the total rounded weight of chosen items in a feasible solution of problem $\mathrm{R}$, and let $x^{\prime}=\left(x_{1}^{\prime} ; x_{2}^{\prime}\right)$ denote a feasible solution of problem $\mathrm{R}$ satisfying $r\left(x^{\prime}\right)=r^{*}$. That is, the $x^{\prime}$ is an optimal solution of problem $\mathrm{R}$.

As for problem $\mathrm{Q}$, we are going to consider two partial instance, R-1 and R-2, of an instance of problem R as follows:

\section{Problem R- $i$}

$$
\begin{array}{ll}
\text { minimize } & r_{i}\left(x_{i}\right)=\sum_{k=1}^{n_{i}} w_{i k}^{\mathrm{R}} x_{i k} \\
\text { subject to } & \sum_{k=1}^{n_{i}} w_{i k} x_{i k} \geq b_{i}, \\
& x_{i k} \in\{0,1\}, k=1,2, \ldots, n_{i} .
\end{array}
$$

Let

$$
W_{i}^{\mathrm{R}}=\sum_{k=1}^{n_{i}} w_{i k}^{\mathrm{R}}, \quad i=1,2,
$$

and let

$$
W^{\mathrm{R}}=W_{1}^{\mathrm{R}}+W_{2}^{\mathrm{R}}
$$

Then, from Eqs. (13) and (14), we have

$$
W^{\mathrm{R}} \leq W / \delta \leq n t / \delta=O\left(\frac{n^{2}}{\varepsilon}\right) .
$$

Problem $\mathrm{R}-i$ is the minimum knapsack problem and also for the problem, there is a pseudo-polynomial time dynamic programming procedure ${ }^{(8,10,11)}$. By using the dynamic programming procedure with a small modification, as Lemma 1, we also have the following lemma:

Lemma 2. For an instance of problem R- $i$, a maximal subset $X_{i}^{\mathrm{R}}$ of feasible solutions of the problem such that any two feasible solutions in the $X_{i}^{\mathrm{R}}$ have different objective function values can be obtained in $O\left(n_{i} W_{i}^{\mathrm{R}}\right)=O\left(n^{3} / \varepsilon\right)$ time.

\section{Heuristic Performance}

\subsection{Algorithm}

Let $m_{i}=\left|X_{i}^{\mathrm{R}}\right|$ denote the number of feasible solutions contained in the maximal set $X_{i}^{\mathrm{R}}$ (see Lemma 2). Note that $m_{i}=O\left(W_{i}^{\mathrm{R}}\right)=O\left(n^{2} / \varepsilon\right)$, since the number of distinct objective function values $r_{i}\left(x_{i}\right)$ to be taken by a feasible solution $x_{i}$ of problem $\mathrm{R}-i$ is at most $W_{i}^{\mathrm{R}}+1$. The feasible solutions $\tilde{x}_{i}^{(h)}\left(h=1,2, \ldots, m_{i}\right)$ in the set $X_{i}^{\mathrm{R}}$ have been ordered without an additional sorting as

$$
r_{i}\left(\tilde{x}_{i}^{(1)}\right)<r_{i}\left(\tilde{x}_{i}^{(2)}\right)<\cdots<r_{i}\left(\tilde{x}_{i}^{\left(m_{i}\right)}\right),
$$

if we apply the pseudo-polynomial time dynamic programming procedure for the minimum knapsack problem.
Of course, it holds $f_{i}\left(\tilde{x}_{i}^{(h)}\right) \geq b_{i}$ for each $h=1,2, \ldots, m_{i}$. For each item type $i \in\{1,2\}$, since the optimal solution $x^{*}=\left(x_{1}^{*} ; x_{2}^{*}\right)$ meets $f_{i}\left(x_{i}^{*}\right) \geq b_{i}$, there is some $h^{\prime} \in\{1,2$, $\left.\ldots, m_{i}\right\}$ such that it satisfies $r_{i}\left(\tilde{x}_{i}^{\left(h^{\prime}\right)}\right)=r_{i}\left(x_{i}^{*}\right)$ from the maximality of the set $X_{i}^{\mathrm{R}}$.

As the next step, the proposed heuristic algorithm in this paper finds a $0-1$ vector $x^{\prime}$ which is a combination of two partial solutions $\tilde{x}_{1} \in X_{1}^{\mathrm{R}}$ and $\tilde{x}_{2} \in X_{2}^{\mathrm{R}}$ such that it meets the target weight constraint $f\left(x^{\prime}\right) \geq t$, and for any other combination $x \in X_{1}^{\mathrm{R}} \times X_{2}^{\mathrm{R}}$ with $f(x) \geq t$, it satisfies $r\left(x^{\prime}\right) \leq r(x)$. Hence, the solution $x^{\prime}$ is a feasible and an optimal solution of problem $\mathrm{R}$. The $x^{\prime}$ is returned as a heuristic feasible solution of problem Q.

The proposed heuristic algorithm is summarized as follows:

\section{Algorithm 1}

Step 1 . For each type $i \in\{1,2\}$ of items, compute a maximal subset $X_{i}^{\mathrm{R}}$ of feasible solutions of problem R- $i$.

Step 2. Find a combination $x^{\prime}=\left(\tilde{x}_{1} ; \tilde{x}_{2}\right)$ of two partial solutions $\tilde{x}_{1} \in X_{1}^{\mathrm{R}}$ and $\tilde{x}_{2} \in X_{2}^{\mathrm{R}}$ such that the $x^{\prime}$ meets the target weight constraint $f\left(x^{\prime}\right) \geq t$, and for any other combination $x \in X_{1}^{\mathrm{R}} \times X_{2}^{\mathrm{R}}$ with $f(x) \geq t$, it satisfies $r\left(x^{\prime}\right) \leq r(x)$.

Step 3. Return $x^{\prime}$.

In Step 1, we obtain two maximal sets $X_{1}^{\mathrm{R}}$ and $X_{2}^{\mathrm{R}}$ in Lemma 2, and the first step requires $O\left(n^{3} / \varepsilon\right)$ time. We then compute a combination of two partial solutions in the two maximal sets $X_{1}^{\mathrm{R}}$ and $X_{2}^{\mathrm{R}}$ by Step 2. Since the partial solutions in the sets $X_{1}^{\mathrm{R}}$ and $X_{2}^{\mathrm{R}}$ have already been sorted (see Eq. (22)), we can utilize an application of the well known linear search technique ${ }^{(5)}$ to find a required combination. It takes $O\left(n^{2} / \varepsilon\right)$ time, since $m_{i}=\left|X_{i}^{\mathrm{R}}\right|=O\left(n^{2} / \varepsilon\right)$. The final step clearly runs in $O(n)$ time. Therefore, the time complexity of Algorithm 1 is evaluated as $O\left(n^{3} / \varepsilon\right)$, which is polynomial in $n$ and $1 / \varepsilon$.

\subsection{Analysis}

Finally, we are going to prove the following theorem:

Theorem 1. For an instance of problem Q, let $f^{*}$ denote the minimum of the total weight, and for the corresponding instance of problem $\mathrm{R}$ with a real $\varepsilon>0$, let $x=x^{\prime}$ denote a feasible solution of problem Q obtained by Algorithm 1 with the time complexity of $O\left(n^{3} / \varepsilon\right)$. Then, it holds

$$
t \leq f\left(x^{\prime}\right) \leq(1+\varepsilon) f^{*} .
$$

Proof. The solution $x^{\prime}$ has been seen to be feasible in the sense of problem Q. We then need to show $f\left(x^{\prime}\right) \leq(1+\varepsilon) f^{*}$.

Again recall that two problems $\mathrm{Q}$ and $\mathrm{R}$ have the same constraints, and hence a feasible solution of problem $\mathrm{Q}$ is also a feasible solution of problem $\mathrm{R}$, and vice versa. Since the $x^{\prime}$ is an optimal solution of problem $\mathrm{R}$, and the $x^{*}$ is a feasible 
solution of problem $\mathrm{R}$, it holds $r\left(x^{\prime}\right) \leq r\left(x^{*}\right)$, i.e.,

$$
\sum_{i=1}^{2} \sum_{k=1}^{n_{i}} w_{i k}^{\mathrm{R}} x_{i k}^{\prime} \leq \sum_{i=1}^{2} \sum_{k=1}^{n_{i}} w_{i k}^{\mathrm{R}} x_{i k}^{*} .
$$

Together with Eq. (13), we obtain

$$
\begin{aligned}
f\left(x^{\prime}\right) & =\sum_{i=1}^{2} \sum_{k=1}^{n_{i}} w_{i k} x_{i k}^{\prime}<\sum_{i=1}^{2} \sum_{k=1}^{n_{i}}\left(\delta w_{i k}^{\mathrm{R}}+\delta\right) x_{i k}^{\prime} \\
& =\delta \sum_{i=1}^{2} \sum_{k=1}^{n_{i}} w_{i k}^{\mathrm{R}} x_{i k}^{\prime}+\delta \sum_{i=1}^{2} \sum_{k=1}^{n_{i}} x_{i k}^{\prime} \\
& \leq \delta \sum_{i=1}^{2} \sum_{k=1}^{n_{i}} w_{i k}^{\mathrm{R}} x_{i k}^{*}+\delta \sum_{i=1}^{2} \sum_{k=1}^{n_{i}} x_{i k}^{\prime} \\
& \leq \sum_{i=1}^{2} \sum_{k=1}^{n_{i}} w_{i k} x_{i k}^{*}+\delta \sum_{i=1}^{2} \sum_{k=1}^{n_{i}} x_{i k}^{\prime} \\
& =f^{*}+\delta \sum_{i=1}^{2} \sum_{k=1}^{n_{i}} x_{i k}^{\prime} \leq f^{*}+\delta n=f^{*}+\varepsilon t \\
& =(1+\varepsilon) f^{*},
\end{aligned}
$$

which completes the proof.

\section{Concluding Remarks}

In this paper, we considered a combinatorial optimization model of mixture packaging of two types of items, which arises in actual food packing systems, so-called multi-head weighers. The total weight of chosen items for a package is asked to be minimized under the condition that the total weight must be no less than a specified target weight. For a package, the weight sum of chosen items of each type must also be no less than a prescribed necessity minimum. In this paper, we designed a heuristic algorithm for the problem of minimizing the total weight, and showed that for a given positive real $\varepsilon$, the time complexity is polynomial in $n$ and $1 / \varepsilon$, where $n$ is the number of all the current items, and the performance factor is $(1+\varepsilon)$.

For future research, it is left for us to incorporate a recursive of computing the total priority of chosen items as the second objective with the proposed heuristic algorithm, in order to reduce the durations of remaining items in hoppers ${ }^{(5)}$, and to examine the empirical performance. Also, the time complexity of the proposed heuristic algorithm may be improved by regarding an upper bound on the optimal weight sum (i.e., the $f_{i}\left(x_{i}^{*}\right)$ ) of chosen items of each type. Further, it would be interesting to examine a performance guarantee of a heuristic algorithm on the total weight of the mixture packaging problem with more than two types of items ${ }^{(6)}$.

\section{Acknowledgment}

This research was partially supported by JSPS KAKENHI Grant JP16K01241.

\section{References}

(1) Morinaka, H., Automatic combination weigher for product foods (in Japanese), Journal of the Japan Society of Mechanical Engineers, Vol. 103, No. 976, (2000), pp. 130-131.

(2) Mechanical Engineering Heritage No. 89: Multi-head weigher ACW-M-1 (in Japanese), Journal of the Japan Society of Mechanical Engineers, Vol. 120, No. 1185, (2017), pp. 32-33.

(3) Wurdemann, H.A., Aminzadeh, V., Dai, J.S., Reed, J., and Purnell, G., Category-based food ordering processes, Trends in Food Science \& Technology, Vol. 22, (2011), pp. 14-20.

(4) Imahori, S., Karuno, Y., Nagamochi, H., and Wang, $\mathrm{X}$., Kansei engineering, humans and computers: Efficient dynamic programming algorithms for combinatorial food packing problems, International Journal of Biometrics, Vol. 3, No. 3, (2011), pp. 228-245.

(5) Imahori, S., Karuno, Y., and Tateishi, K., Dynamic programming algorithms for producing food mixture packages by automatic combination weighers, Journal of Advanced Mechanical Design, Systems, and Manufacturing, Vol. 8, No.5, (2014), [DOI: 10.1299/ jamdsm.2014jamdsm0065].

(6) Imahori, S., Karuno, Y., and Tateishi, K., Pseudopolynomial time algorithms for combinatorial food mixture packing problems, Journal of Industrial and Management Optimization, Vol. 12, No. 5, (2016), pp. 10571073.

(7) Karuno, Y. and Nakahama, O., Greedy heuristic performance for combinatorial mixture packaging of two types of foods with bounded weights, Proceedings of the Ninth International Conference on Leading Edge Manufacturing in 21st Century, (LEM21 Hiroshima), (2017), C11, pp. 1-6.

(8) Vazirani, V.V., Approximation Algorithms, Second Ed., (2001), Springer, Berlin.

(9) Ibaraki, T., Algorithms and Data Structures in $C$ (in Japanese), (1999), Shoko-do, Tokyo.

(10) Karuno, Y. and Saito, R., Heuristic algorithms with rounded weights for a combinatorial food packing problem, Journal of Advanced Mechanical Design, Systems, and Manufacturing, Vol. 11, No. 1, (2017), [DOI: 10.1299/jamdsm.2017jamdsm0003].

(11) Asano, T. and Imai, H., Computation and Algorithms (in Japanese), (2000), Ohmsha, Tokyo. 